\title{
Anemia em alunos de escolas públicas no Recife: um estudo de ten dências temporais
}

\author{
Anemia in students at public schools in Recife: \\ a study of temporal trends
}

\author{
M aria da Conceição Chaves de Lemos ${ }^{1}$ \\ I da Cristina Ferreira Leite ${ }^{2}$ (In memorian) \\ Juliana Souza Oliveira ${ }^{3}$ \\ Teresa Cristina M iglioli ${ }^{2}$ \\ M arcela Carvalho dos Santos ${ }^{2}$ \\ M alaquias Batista Filho ${ }^{2}$
}

\footnotetext{
${ }^{1}$ Hospital Agamenon M agalhães. Estrada do Arraial 2723, Casa Amarela. CEP 52051-380 Recife PE. chavesdelemos@uol.com.br

${ }^{2}$ Instituto M edicina Integral Professor Fernando Figueira - IMIP - Recife/PE

${ }^{3}$ Centro Acadêmico de Vitória, Universidade Federal dePernambuco UFPE
}

Abstract The scope of this study was to evaluate trends in the temporal evolution of anemia in students ranging from theages of 7 to 11 in Várzea district (Recife-PE) based on three reports concluded in 1982, 2001 and 2005. An evaluation was achieved in ten public schools in the first study and nine in the last two (one was shut down), in a sample of 305 students (1982), 684 students (2001) and 756 students (2005). H emoglobin was determined in blood samples taken by venopunction, having two criteria to describe anemia: cut points $<12 \mathrm{~g} / \mathrm{dL}$ and $<11.5 \mathrm{~g} / \mathrm{dL}$. By the first criteria, the prevalence of anemia increased from $8.8 \%$ in 1982 to $18.9 \%$ in 2001 , decreasing to $13.4 \%$ in 2005 . In the last evaluation, the problem of anemia practically disappeared starting from the age of 9 . The evolution of anemia revealed two very different trends: marked elevation in the first phase (1982 to 2001) and a rapid decreasein the second stage (2001 to 2005). Key words Anemia, Scholar health, Crosssectional studies
Resumo 0 objetivo deste estudo foi avaliar as tendências de evolução temporal da anemia em alunos de 7 a 11 anos no bairro da Várzea (Recife- $P E$ ) com base em três inquéritos realizados em 1982, 2001 e 2005. A avaliação foi efetuada em dez escolas públicas no $1^{\circ}$ estudo e nove nos dois últimos (uma foi desativada), em amostra de 305 alunos (1982), 684 alunos (2001) e 756 alunos (2005). A hemoglobina foi determinada em amostras de sangue col hidas por venopunção, considerando dois critérios para discriminar a anemia: pontos de corte $<12 \mathrm{~g} / \mathrm{dL}$ e $<11,5 \mathrm{~g} / \mathrm{dL}$. Pelo primeiro critério, a prevalência de anemia el evou-se de 8,8\% em 1982 para 18,9\% em 2001, decaindo para $13,4 \%$ em 2005. Pel o segundo critério, a ocorrência de anemia decresceu de $10,7 \%$ para 3,6\%, entre 2001 e2005. N a última avaliação, o problema da anemia praticamente desapareceu, a partir dos 9 anos de idade. A evolução da anemia apresentou duas tendências bem diferenciadas: el evação num primeiro momento (1982 - 2001) e rápida diminuição numa segunda etapa (20012005)

Palavras-chave Anemia, Saúde escolar, Estudos transversais 
Introdução

Uma das singularidades da epidemiologia nutricional do Brasil nas últimas três décadas (a partir de 1974-75) consistenas evidências deagravamento da prevalência deanemia em crianças menores de cinco anos $\mathrm{s}^{1,2,3} \mathrm{em}$ contraste com a substancial redução da desnutrição energético-proteica(DEP), da deficiência deiodo e, possivel mente, da hipovitaminose $\mathrm{A}^{4,5}$. Ao mesmo tempo, define-seo processo pandêmico etrans-social do sobrepeso/obesidade na população adulta, praticamente triplicando sua ocorrência nos últimos 30 anos $5^{6,7}$. No caso específico da anemia, apesar da escassez de estudos, pesquisas real izadas no município de São Paulo ${ }^{2}$ eno estado da Paraíba ${ }^{3}$ descrevem aumentos na prevalência quevariam de $88 \%$ a $213 \%$, no decurso de 10 a 21 anos entre os estudos comparados. 0 exemplo mais ilustrativo destas tendências seria o caso da cidade de São Paulo, onde a ocorrência de anemia em menores de cinco anos aumentou de $22 \%$ (1974/75) para 35\% (1984/85), elevando-se para $46,9 \%$ em 1995/962. Resultados pontuais obtidos em avaliações de al gumas capitais e grandes cidades do Brasil na segunda metade da década de 90 retratam valores bem seme Ihantes aos descritos para a cidade de São Paulo (1995-96), sugerindo queten dências semelhantes poderiam ser reproduzidas em outras regiões do país ${ }^{2,8,9}$.

$\mathrm{Na}$ realidade, trabalhos publicados no Brasil ${ }^{10}$ e em vários outros países, bem como relatórios da Organização Mundial da Saúde ${ }^{11}$ e da Organização Panamericana de Saúde ${ }^{12}$, são concordantes em observar que a anemia representa, em termos de magnitude, a principal endemia carencial dos tempos modernos, afetando, em diferentes graus de ocorrência, todos os segmentos biológicos e estratos socioeconômicos. Face a esta situação, a partir de 1990, as Nações Unidas passaram a incluir formalmente a anemia como uma das prioridades de saúde e nutrição, com a proposição de metas internacionais para seu controle $e^{13}$. No caso do Brasil, diante de evidências de que a anemia estaria configurando uma endemia de caráter progressivo, o M inisté rio da Saúde adotou medidas de caráter setorial (utilização de fármacos de ferro) e de natureza mais genérica (enriquecimento dealimentos com ferro e folato), tentando reverter as tendências do agravamento do problema, com ênfase na área materno-infantil ${ }^{14,15}$. N este contexto, um segmento populacional ainda pouco estudado (crianças em idade escolar) passa a representar um interessepeculiar, pelos efeitos potencial mente adversos da anemia no processo de aprendizagem $^{16}$. Ademais, o Programa Nacional de Alimentação do Escolar (PNAE) representa o mais antigo, mais extenso e mais consolidado programa denutrição no Brasil, cobrindo uma clientela de 34,6 milhões de beneficiários, o quelheconferetambém, ser considerado um dos maiores programas na área de alimentação escolar no mundo, sem se dispor de uma avaliação satisfatória sobre seu estado nutricional17-20.

Sob o aspecto metodológico interessa analisar os resultados derivados da inclusão de dois pontos de corte para a discriminação da anemia, em face das novas recomendações da OM S ${ }^{11,21}$. Isto se justifica sob o ponto de vista conceitual, isto é, a observação de que os dois pontos de corte podem alterar substancialmente os resultados e, ainda, pela impossi bilidade operacional de aplicar o novo ponto de corte aos dados de 1982, conforme será explicado na metodologia.

0 artigo considera dois objetivos básicos: a) avaliar a prevalência e as tendências de evolução temporal da anemia em escolares dos mesmos estabelecimentos de ensino estudados no município do Recifenosúltimos 23 anos (198222, $2001^{23}$, 200524); b) analisar, comparativamente, as prevalências da anemia referentes à aplicação de dois pontos de corte, considerando o critério mais antigo $(<12 \mathrm{~g} / \mathrm{dL})$ e o mais recente $(<11,5 \mathrm{~g} / \mathrm{dL})$.

\section{Metodologia}

Tratando-se detrês inquéritos descritivos do tipo transversal, realizados nas mesmas escolas em tempos diferentes $\left(1982^{22}, 2001^{23}, 2005^{24}\right)$, com objetivos múltiplos (um dos quais a avaliação da anemia), num contexto de marcantes mudanças da situação nutricional do país, e efetuadas por pesquisadores independentes, as estimativas amostraisesua operacionalização implicaram em procedimentos diferentes, conforme se descreve a seguir:

a) Nos três inquéritos, o intervalo de confiança (95\%) e a precisão de prevalência de 3\% foram as mesmas, para universos de 2.413 alunos (1982), 2.000 alunos (2001) e 2.200 alunos (2005).

b) As prevalências esperadas para a ocorrência de anemia foram de $9 \%$ ( $1^{\circ}$ estudo), $20 \%$ ( $2^{\circ}$ estudo) e $25 \%$ ( $3^{\circ}$ estudo) com base em pesquisas praticamente contemporâneas realizadas em escolares de outras localidades do N ordeste.

c) Em relação aos procedimentos: no primeiro estudo (1982) foram sorteados um de cada 
seis alunos cujos pais concordaram em sua participação na pesquisa, resultando numa amostra de 305 casos; no segundo e terceiro estudo, sobre a amostra basal aplicou-se uma margem de segurança de $30 \%$, resultando, finalmente, numa amostra estudada de 684 alunos (ano 2001) e 756 (ano 2005).

Nos dois primeiros estudos, os teores de he moglobina, foram determinados pela técnica da cianometahemoglobina ${ }^{21}$, mediante fotocolorimetria, em amostras de sangue venoso do antebraço, com os alunos observando um jejum noturno de oito a 12 horas. $\mathrm{Na}$ última avaliação, a hemoglobina foi determinada por nova técnica colorimétrica aprovada pela American Society for Clinical Patology25, mantendo-se os demais procedimentos dos estudos anteriores.

Os resultados foram dicotomizados em casos normais e anemia, utilizando-se dois pontos de corte: 0 antigo, discriminado pelo limite de 12 $\mathrm{g} / \mathrm{dL}$ de hemoglobina21, e o critério mais atual proposto pela Organização Mundial da Saúde ${ }^{11}$ considerando o valor de $11,5 \mathrm{~g} / \mathrm{dL}$. Assim, a série temporal reunindo os três estudos (1982, 2001 e 2005) se constituiu ten do como elemento comum de comparação o ponto decorte mais antigo (12g/ dL). Como os dados de 1982 não estão mais disponíveis como variáveis individualizadas, mas apenas como valores agregados, tornando-se impossível a aplicação retrospectiva do novo ponto de corte. Já os estudos de 2001 e 2005 podem ser analisados utilizando também o novo ponto de corte de $11,5 \mathrm{~g} / \mathrm{dL}$, que passou a vigorar a partir de $1998^{11}$.

Para as decisões estatísticas das hipóteses de diferenças, foram utilizados os seguintes testes, devidamente explicitados nas tabelas de resultados: qui-quadrado, M cNemar e o teste de Wald para tendência. Nas provas estatísticas foi adotado o nível de significância de 5\%. Para processamento e análise dos dados estatísticos foram utilizados os softwares Epi-Info 6.04 eStata 9.2.

Por representar uma consolidação detrês pesquisas distintas, os estudos de campo receberam encaminhamentos diferentes, sob o aspecto ético. No primeiro (1982) quando o Brasil ainda não dispunha de normas formais sobre pesquisas em seres humanos, por iniciativa e orientação do grupo de investigadores, os alunos foram examinados mediante assinatura do protocolo de concordância livre e esclarecido, diretamente entre gue pelos professores de cada sala de aula aos pais ou responsáveis legais dos alunos. $N$ as duas pesquisas subsequentes, além das cartas de consentimento, os respectivos projetos foram devi- damente aprovados nas instâncias competentes: o estudo de 2001 pelo Comitê de Ética do Centro de Ciências da Saúde da Universidade Federal de Pernambuco, enquanto o estudo de 2005 foi autorizado pelo Comitê de Ética do Hospital Agamenon Magalhães. Os casos de anemia detectados nas pesquisas foram tratados com sulfato ferroso, segundo normas padronizadas.

\section{Contexto do estudo}

No estudo inicial (1982)22, a justificativa da escolha do bairro da Várzea como área de estudo da situação alimentar e nutricional dos escolares, apoiava-se na existência de um projeto docente-assistencial da Universidade Federal de Pernambuco - UFPE, na disponibilidade de uma rede de serviços públicos de saúde e de educação dos governos federal, estadual e municipal, que poderia ser mobilizada para uma ação conjunta, em função de um projeto de educação nutricional liderado pelo Departamento de Nutrição da UFPE e, ainda, pela heterogeneidade de condições socioeconômicas e ambientais da população. 0 bairro era então habitado por uma classe média baixa, com inúmeros conglomerados de áreas faveladas. $\mathrm{No}$ ano de 2000, a Várzea tinha uma população de 30.092 habitantes, apresentando, segundo o Programa das Nações Unidas para o Desenvolvimento ${ }^{26}$, um Índice de Desenvolvimento Humano (IDH) entre 0,740 e 0,765 em suas três subáreas socioeconômicas. Considera-se que este bairro seria representativo do mosai co de desigualdades do Recife, retratado no estudo clássico de Gilberto Freire (Sobrados e M ocambos) e atualizado no Atlas do Desenvolvimento Humano do Recife ${ }^{26}$. N a avaliação mais recente, a taxa de analfabetismo dos adultos ( 25 anos e mais residentes no bairro) era de $16,9 \%$, 96\% das crianças de 7 a 14 anos frequentavam a escola, 90\% dos domicílios dispunham de água encanada, o índice de mortalidade infantil era de 35 por 1.000 nascidos vivos e a expectativa de vida alcançava 66 anos.

$\mathrm{Na}$ área de estudo funcionavam, em 1982, dezenove escolas, com 2.900 al unos de setea onze anos de idade, matriculados da 1aa a 4á série, dos quais $64 \%$ matriculados em seis escolas estaduais e 30\% em seis escolas municipais; enquanto os $6 \%$ restantes estudavam em sete escolas particulares. Na primeira avaliação (1982) ${ }^{22}$ foram incluídas na amostragem dez escolas públicas: seis estaduais e quatro do município. Das 10 escolas públicas estudadas em 1982, uma foi desativada, sendo seus alunos redistribuídos em ou- 
tras unidades de ensino, de modo que, nas avaliações subsequentes (2001 e 2005) ) $^{23,24}$ as escolas reavaliadas foram representadas por nove educandários públicos.

\section{Resultados}

Considerando o ponto de corte de hemoglobina $<12 \mathrm{~g} / \mathrm{dL}$, a prevalência de anemia no total da amostra de escolares aumentou de 8,8\% em 1982 para $18,9 \%$ no ano 2001 , decaindo para $13,4 \%$ em 2005. Esse aumento na frequência de anemia foi estatisticamente significativo, da mesma formaquea diminuição da prevalência do problema nos últimos quatro anos da comparação temporal (2001 e 2005) foi também significativa ( $p$ < $0,001)$. De modo geral, as tendências de evolução temporal do problema para o conjunto da amostra se mantiveram em relação à distribuição entre alunos e alunas, seja em termos de aumento de frequência das prevalências seja de diminuição. No entanto, a variação verificada entre 1982 e2005 no sexo masculino não foi significativa. Em relação à idade, a prevalência da anemia aumentou, em todas as faixas etárias, entre o primeiro e 0 segundo inquérito, diminuindo sistematicamenteno terceiro em relação ao segundo. No entanto, em termos de análises estatísticas, as diferenças temporais nos grupos de 9 e 10 anos, não variaram significativamente (Tabela 1 ).

U tilizando o novo ponto de corte $(11,5 \mathrm{~g} / \mathrm{dL})$, a prevalência da anemia, em 2001 passou a ser de $10,7 \%$, baixando para 3,6\% em 2005. Esta diferençafoi estatisticamentesignificativa $(p<0,001)$.
Os resultados do ano 2001 demonstraram uma distribuição bastante semelhante na frequência da anemia por grupos etários, variando entre $9,5 \%$ e $12,9 \%$. Já na última avaliação (2005) a ocorrência de anemia apresentou-se mais elevada (acima de 7\%) nos dois primeiros grupos de idade, decaindo para valores próximos a $1 \%$ a partir de 9 anos. Com exceção dos dois primeiros grupos, em todas as outras idades as reduções de prevalência entre 2001 e 2005 foram significativas (Tabela 2).

A Tabela 3 apresenta as prevalências de anemia dos dois últimos inquéritos com a utilização dos dois critérios de definição de anemia (pontos de corte $11,5 \mathrm{~g} / \mathrm{dL}$ versus $12,0 \mathrm{~g} / \mathrm{dL}$ ), bem como, as diferenças resultantes dos dois momentos da pesquisa: 2001 e2005. As diferenças foram estatisticamentesignificativas, seja em relação aos resultados comparativos dos métodos de classificação entre si, seja na variação resultante do mesmo critério em função dos dois momentos sequenciais da avaliação.

\section{Discussão}

Em função dos objetivos do estudo, duas observações bem distintas devem ser ressaltadas na análise dos resultados: a) entre 1982 e 2001, 0 problema da anemia se elevou de forma marcante, passando de uma situação inicial de 8,8\% para $18,9 \%$, tendo como referência o antigo ponto decortede hemoglobina em $12 \mathrm{~g} / \mathrm{dL}$. I sto correspondea uma elevação cumulativa anual deaproximadamente $0,5 \%$, mais do que duplicando a

Tabela 1. Prevalência de anemia em alunos de escolas públicas segundo o ponto de corte de hemoglobina abaixo de 12 g/dL. Recife, 1982, 2001 e 2005.

\begin{tabular}{|c|c|c|c|c|c|c|c|}
\hline \multirow{2}{*}{ Especificações } & \multicolumn{2}{|c|}{1982} & \multicolumn{2}{|c|}{2001} & \multicolumn{2}{|c|}{2005} & \multirow[b]{2}{*}{ Valor $\mathrm{p}^{*}$} \\
\hline & $n$ & $\%$ & $n$ & $\%$ & $\mathrm{n}$ & $\%$ & \\
\hline \multicolumn{8}{|l|}{ Sexo } \\
\hline M asculino & 135 & 9,6 & 310 & 15,5 & 362 & 13,8 & 0,246 \\
\hline Feminino & 170 & 8,2 & 374 & 21,7 & 394 & 12,9 & $<0,001$ \\
\hline Total & 305 & 8,8 & 684 & 18,9 & 756 & 13,4 & $<0,001$ \\
\hline \multicolumn{8}{|l|}{ Idade (anos) ${ }^{t}$} \\
\hline 7 & 41 & 4,9 & 119 & 23,5 & 140 & 21,4 & 0,014 \\
\hline 8 & 68 & 8,8 & 139 & 23,0 & 152 & 15,8 & 0,002 \\
\hline 9 & 72 & 8,3 & 148 & 17,6 & 161 & 16,2 & 0,184 \\
\hline 10 & 70 & 8,6 & 137 & 15,3 & 174 & 8,0 & 0,097 \\
\hline 11 & 54 & 13,0 & 141 & 15,6 & 129 & 5,4 & 0,026 \\
\hline Total & 305 & 8,8 & 684 & 18,9 & 756 & 13,4 & $<0,001$ \\
\hline
\end{tabular}

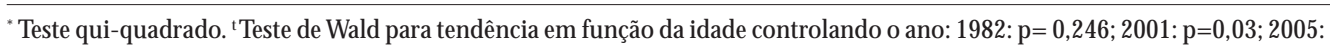
$p=<0,001$ 
Tabela 2. Prevalência de anemia em alunos de escolas públicas segundo o ponto de corte de hemoglobina abaixo de 11,5 g/dL. Recife 2001 e 2005.

\begin{tabular}{lrrrrrrr}
\hline \multirow{2}{*}{ Especificações } & \multicolumn{2}{c}{2001} & & \multicolumn{2}{c}{2005} & \\
\cline { 2 - 3 } & $\mathrm{n}$ & $\%$ & & $\mathrm{n}$ & $\%$ & Valor $\mathrm{p}^{*}$ \\
\hline Sexo & 310 & 9,4 & & 362 & 2,8 & $<0,001$ \\
$\quad$ M asculino & 374 & 11,8 & & 394 & 4,3 & $<0,001$ \\
$\quad \begin{array}{l}\text { Feminino } \\
\quad \text { Total }\end{array}$ & 684 & 10,7 & & 756 & 3,6 & $<0,001$ \\
$\begin{array}{l}\text { Idade (anos) } \\
7\end{array}$ & 119 & 10,9 & & 140 & 7,1 & 0,286 \\
8 & 139 & 12,9 & & 152 & 7,9 & 0,157 \\
9 & 148 & 9,5 & & 161 & 0,6 & $<0,001$ \\
10 & 137 & 9,5 & & 174 & 1,1 & 0,001 \\
11 & 141 & 10,6 & & 129 & 1,6 & 0,002 \\
Total & 684 & 10,7 & & 756 & 3,6 & $<0,001$ \\
\hline
\end{tabular}

Testedo qui-quadrado.

prevalência da anemia no período de 19 anos; b) num segundo momento (entre 2001 e 2005) registrou-se uma diminuição expressiva na frequência do problema, representando um decréscimo linear de praticamente $1,4 \%$ por ano. Assim, o ritmo de redução na fase mais recentefoi quase três vezes superior ao ritmo anual deincremento da endemia, que teria evoluído da categoria leve (menos de 10\% na década de 90 ) para a condição moderada, no início dos anos 2000, segundo critérios de classificação epidemiológica dos níveis de prevalência ${ }^{11}$.

Na mesma linha de análise ao se utilizar 0 segundo ponto de corte ( $11,5 \mathrm{~g} / \mathrm{dL})$, a sequência temporal, por motivos já explicados, se limita aos estudos de 2001 e 2005. Repetindo a tendência anterior, observou-se acentuado declínio na prevalência de anemia. M ais do que a significação estatística da comparação, é muito relevante assinalar queas frequências encontradas em 2005 seriam compatíveis com a condição epidemiológica de controle da anemia, considerando que a ocorrência de $3,6 \%$ já não configura um problema de saúde coletiva, segundo critério da OM $\mathrm{S}^{11}$. Em estudo semelhante, em Brasília, Heijblom e Santos ${ }^{27}$, verificaram quea ocorrência de anemia em 427 alunos de 6 a 11 anos aumentou de $21,5 \%$ para 26,9\%, entre 1984 e 2004, adotando-se 0 ponto de corte de $12 \mathrm{~g} / \mathrm{dL}$. Desafortunadamente, não se dispõe de outros estudos semel hantes para aprofundar as análises comparativas sobre estatendência.

Em relação ao estudo realizado em escolas do Recife, no ano de 2005, a distribuição dos resulta-
Tabela 3. Prevalência de anemia em alunos de escolas públicas segundo dois pontos de corte dehemoglobina. Recife, 2001 e 2005.

\begin{tabular}{lrrrrrrr}
\hline Especificações & \multicolumn{2}{c}{2001} & & \multicolumn{2}{c}{2005} & & \\
\cline { 2 - 3 } & $\mathrm{n}$ & $\%$ & & $\mathrm{n}$ & $\%$ & Valor $\mathrm{p}^{*}$ \\
\hline Pontos de corte & & & & & & \\
$\quad<11,5 \mathrm{~g} / \mathrm{dL}$ & 684 & 10,7 & 756 & 3,6 & $<0,001$ \\
$<12,0 \mathrm{~g} / \mathrm{dL}$ & 684 & 18,9 & 756 & 13,4 & 0,006 \\
$\quad$ Valor $\mathrm{p}\left({ }^{* *}\right)$ & & $<0,001$ & & $<0,001$ &
\end{tabular}

(*) Teste do qui-quadrado; $\left.{ }^{(*)}\right)$ Teste de M cNemar

dos por faixas etárias, apenas nos grupos de 7 e 8 anos a anemia aparece como um problema residual, com ocorrências abaixo de $8 \%$, enquanto nas demais idades a frequência variou em torno de $1,1 \% \pm 0,5 \%$. Ademais, apenas nestes dois grupos, a redução da anemia não alcança significação estatística em relação aos resultados de 2001. Trata-se de um resultado surpreendentemente baixo em relação aos relatos sobre o problema no Brasi| ${ }^{27,28}$ e até mesmo em escala mundial ${ }^{29}$.

Por se tratar do único estudo de caráter temporal efetuado no Brasil em alunos das mesmas escolas públicas, seus resultados tornam-se singularmenterelevantes, evidenciando, num primeiro momento, uma tendência que já havia sido registrada em menores decinco anos em São Paulo entre 1974/75 e 1995/962 eno Estado da Paraíba, entre 1982 e 1992, por Oliveira et al ${ }^{3}$. Expressas em médias de variações percentuais, as mudanças de prevalência em crianças do município de São Paulo no período 1974/75 e 1995/96, representariam um aumento anual de $1,2 \%$, mais do que duplicando sua ocorrência nos extremos da observação temporal: de $22 \%$ para $46,9 \%$. Aplicando-se o mesmo procedimento nas avaliações efetuadas em menores de cinco anos no estado da Paraíba ${ }^{3}, 0$ aumento corresponde, praticamente, a $1 \%$, ao ano, bem aproximado, portanto, do que se verificou na cidade de São Paulo².

Numa visão simplificada, este estudo revelaria, portanto, duas tendências antagônicas: uma primeira, de aumento de prevalência de anemia em escolares, correspondendo ao que fora descrito em menores de cinco anos ${ }^{2,3}$; uma segunda tendência, em sentido contrário, num ritmo surpreendentemente el evado, de rápido declínio do problema.

M esmo sem dispor de uma explicação empiricamente demonstrada, é muito provável quea 
rápida mudança de situação, neste segundo momento, na prevalência da anemia em escolares da Várzea passa a ser atribuída, em grande parte, aos efeitos da fortificação, a partir de 2004, das massas alimentares de consumo elevado, ou, explicitamente, das farinhas de trigo e de milho, tornada obrigatória pela Resolução da Diretoria Colegiada (RDC) 344 da Agência Nacional de Vigilância Sanitária (ANVISA) de 13/12/200214. Esta medida consistiu na incorporação de 4,2 mg de ferro elementar e $150 \mu \mathrm{g}$ de ácido fólico para cada 100 gramas de farinha de trigo e de milho. Admitindo-se como o fator mais provável do declínio da anemia, isto significaria que a redução anual do problema, a partir de 2001, teria sido de 5,5\% em termos de diferenciais relativos. No entanto, considerando apenas um ano a partir da aplicação efetiva da fortificação das massas alimentares (junho de 2004), a conclusão se ria que este impacto teria ocorrido já no primeiro ano de aplicação da medida. Ao se considerar como referência o segundo ponto de corte para definição de anemia (abaixo de 11,5 g/dL) o resultado seria ainda mais expressivo, desde que a prevalência mais recente (2005) de 3,6\% indicaria que o problema já se acha sob controle em termos epidemiológicos. Deve-se considerar que o uso de produtos industrializados derivados de trigo ou milho, como os biscoitos, representam um dos itens de consumo alimentar que mais aumentaram no Brasil, em anos recentes ${ }^{30}$.

A propósito, é oportuno referir que, em São Bernardo do Campo ${ }^{31}$, um estudo especificamente desenhado para avaliar o efeito da fortificação do ferro na alimentação escolar, observou que esta medida teria aumentado em $22 \%$ a biodisponibilidade do ferro nas refeições servidas na escola, contribuindo para baixar a prevalência da ane mia para $5,7 \%$. Ora, se for considerado que a alimentação na escola representa apenas 15 a 30\% de cobertura das necessidades nutricionais dos escolares, eadmitindo-se queno convívio familiar, as massas alimentares entrem habitualmente no cardápio, seria razoável presumir um incremento possivelmente acima de $2 / 3$ (ou $66 \%$ ) na provisão total de ferro biodisponível viabilizado pela estratégia da fortificação obrigatória.

Como parece que a adição de ferro às massas detrigo emilho poderepresentar a interpretação mais real ista paraa mudança ocorrida entre 2001 e 2005 tal como ocorreu em São Bernardo do Campo, os resultados aqui apresentados seriam indicativos da pertinência e relevância da fortificação de alimentos no controle da anemia ferropriva, não apenas em escolares, mas, provavel- mente, nos diversos segmentos da população expostas à anemia ${ }^{12}$. N este sentido, 0 estudo realizado em alunos do bairro da Várzea poderia ter um significado bem mais abrangente.

Outro aspecto a ser considerado consiste no ponto de corte adotado para caracterizar a ocorrência da anemia. Existem questionamentos ponderáveis sobre a acurácia dos padrões hematimé tricos recomendados, seja para separar os casos de anemia, seja para estabelecer sua classificação morfológica efuncional ${ }^{32,33}$. I dade, desfechos esperados, etnia, altitude, estadosfisiológicos, atividades físicas e condições patológicas resultam em variações significativas que não podem ser compreendidas no simples dualismo sim ou não do ponto usado para estabelecer a separação. M esmo sem solucionar estas pendências conceituais e suas implicações, elimite crítico de: $11,5 \mathrm{~g} /$ $\mathrm{dL}$, queatualmenteéa recomen dação convencional para escolares na faixa de idade aqui estudada, ao invés de $12 \mathrm{~g} / \mathrm{dL}$, como seindicava em estudos anteriores, parece um critério bem mais consistente. Adotando-se o novo ponto de corte a prevalência de anemia se modifica substancialmente, seja em relação ao grupo total de escolares estudados, seja em sua distribuição etária na medida em que, por exemplo, o problema da anemia desapareceria a partir dos nove anos de idade.

$N$ a realidade, os resultados deste estudo estabelecem uma oportunidade para se analisar 0 comportamento da anemia em escolares no contexto do processo detransição nutricional no Brasil. As mudanças ocorridas entre 1982 e 2001 na prevalência do problema entre escolares do bairro da Várzea se inscreveram e até serviram de suporte, ao lado de dois outros estudos, para delinear uma tendência diferenciada no quadro de rápidas mudanças no cenário epidemiológico da nutrição no Brasil|2,4. Ou seja, a diminuição da desnutrição energético-proteico (DEP), acompanhada do aumento de ocorrência da anemia em crianças e escolares e do sobrepeso/obesidade em adultos. Esta tendência teria mudado, de forma marcante, no período mais recente de avaliação (2001-2005) tomando-se este estudo em escolares da Várzea o possível indicativo de uma nova situação que estaria se delineando para o país.

Sob o aspecto metodológico, duas observações adicionais parecem pertinentes. Assim, considera-se a limitação de não se poder contar com o banco de dados produzido em 1982, de forma a reavaliar as informações usando o ponto de corte de $11,5 \mathrm{~g} / \mathrm{dL}$. Desta forma, a comparação, com este novo ponto ficou restrita ao período 20012005. Por outro lado, considera-se que o empre- 
go de outro método colorimétrico ${ }^{25}$ para dosagem de hemoglobina, na pesquisa de 2005, praticamente não alteraria a comparabilidade dos resultados, em relação à técnica da cianometahemoglobina ${ }^{21}$ utilizada nos dois estudos anteriores.

Conclusivamente, pode-se estabelecer que o estudo das tendências temporais na ocorrência deanemia em alunos deescolas públicas do bairro da Várzea revela aspectos interessantes e originais da epidemiologia do problema, como a esperada concordância com resultados esperados a partir de estudos em menores de cinco anos, ao lado do rápido einesperado declínio do problema entre os anos 2001 e 2005. Outro resultado muito interessanteseria representado nas grandes mudanças de resultados que se configura na comparação dos pontos de corte ( $<12 \mathrm{~g} /$ $\mathrm{dL}$ versus $<11,5 \mathrm{~g} / \mathrm{dL}$ ) como pontos críticos de definição de anemia. A hipótese de que o enriquecimento de massas alimentares com ferro e folato tenha representado, ao lado de outros fatores, uma possível variável explicativa para a mudança recente na prevalência da anemia, ain- da que colocada em termos de uma evidência preliminar, é um interessante ponto de questionamento para novos estudos.

Já no terceiro estudo a diminuição de prevalência, por quaisquer dos pontos de corte aplicados, foi muito significativa, invertendo-se, num breve espaço de tempo, o curso evolutivo do problema que seria esperado, a partir das tendências dos outros estudos de avaliação histórica. 0 estudo demonstra, ainda, que a simples alteração de um ponto de corte para outro (12 $\mathrm{g} / \mathrm{dL}$ para $11,5 \mathrm{~g} /$ $\mathrm{dL}$ ) implica em uma mudança substancial no nível de ocorrência de anemia. Outro estudo ${ }^{34} \mathrm{em}$ escolares de Maceió demonstrou que usando 0 ponto de corte de $12 \mathrm{~g} / \mathrm{dL}$, a prevalência de anemia erade $25,4 \%$, reduzindo para $9,9 \%$, ao seconsiderar o ponto de corte de $11,5 \mathrm{~g} / \mathrm{dL}$. Estas observações tornam-se importantes nos estudos temporais sobre o problema, impondo-se os necessários ajustes e correções comparativas para contornar o grande viés que pode ser produzido com $0 \mathrm{em}$ prego das novas recomendações da OMS.

\section{Colaboradores}

M CC Lemos, ICF Leite, JS O liveira, TC M iglioli, MC dos Santos e M Batista filho participaram igualmente de todas as etapas de elaboração do artigo.

\section{Referências}

1. Lira PIC, Ferreira LOC. Epidemiologia da anemia ferropriva. In: Kac G, Sichieri R, Gigante DP, organizadores. Epidemiologia Nutricional. Rio de Janeiro: Fiocruz/Atheneu; 2007. p. 297-324

2. Monteiro CA, Szarfarc SC, M ondini L. Tendência secular da anemia na infância na cidade de São PauIo (1984-1996). Rev Saude Publica 2000; 34(Supl.6): 62S-72S.

3. Oliveira RC, Diniz AS, Benigna MJC, Miranda-Silva SM, Lola M M, Gonçalves MC, Asciutti-M oura L, Rivera MA, Santos, LM P. Magnitude, distribuição espacial e tendência da anemia em pré-escolares da Paraíba. Rev Saude Publica 2002; 36(1):26-32.

4. Batista Filho M, Assis AO, Kac G. Transição nutricional: conceitos e características. In: Kac G, Sichieri R, Gigante DP, organizadores. Epidemiologia Nutricional. Rio de Janeiro: Fiocruz/Atheneu; 2007. p. 445-460.

5. West Jr KP. Extent of vitamin A deficiency among preschool children and women of reproductive age. J Nutr 2002; 132:S2857-2866.

6. Anjos LA. O besidade e saúde pública. Rio de Janeiro: Fiocruz; 2006.

7. Monteiro CA, Conde WL, Popkin BM . Incomespecific trends in obesity in Brazil: 1975-2003. Am J Public Health 2007; 97(10):1808-1812. 
8. Batista Filho M, Romani SAM, organizadores. Alimentação, nutrição e saúde no Estado de Pernambuco: espacialização e fatores sócio-econômicos. Recife: Instituto M aterno-Infantil de Pernambuco; 2002. (Série de Publicações Científicas do Instituto Materno-Infantil de Pernambuco, n.7).

9. Assis AM O, Barreto ML, Gomes GSS. Prevalência e fatores associados à ocorrência da anemia em préescolares na cidade de Salvador, Bahia. Cad Saude Publica 2004; 20(6):1633-1641.

10. Batista Filho M, Souza AI, Bresani CC. Anemia como problema de saúde pública: uma realidade atual. Cien Saude Colet 2008; 13(6):1917-1922.

11. World Health Organization (WHO). Iron deficiency anaemia assessment, prevention and control: a guide for programme managers. Geneve; WHO; 2001.

12. Pan American Health Organization (PAHO). Flour fortification with iron, folic acid and vitamin B12. Santiago: PAH 0; 2003.

13. Fundo das Nações Unidas para a Infância (UNICEF). Estratégia para melhorar a nutrição de crianças e mulheres nos países em desenvolvimento: um exame de políticas. New York: UNICEF; 1990.

14. Brasil. Ministério da Saúde, Agência Nacional de Vigilância Sanitária. Resolução - RDC no 344 de 13 de Dezembro de 2002. Aprova o Regulamento Técnico para a Fortificação das Farinhas de Trigo e das Farinhas de Milho com Ferro e Ácido Fólico. Diário Oficial da União 2002; 18 dez.

15. Brasil. Ministério da Saúde. M anual O peracional do Programa Nacional de Suplementação de Ferro. Brasília: M inistério da Saúde; 2005.

16. Pollitt E. Early iron deficiency anemia and later mental retardation. Am J Clin Nutr 1999; 69(1):4-5.

17. Fundo Nacional de Desenvolvimento da Educação (FNDE). Dados estatísticos. [acessado 2009 dez]. Disponível em: http://www.fnde.gov.br/index.php/aedados-estatisticos

18. Burlandy L, Anjos LA. Acesso à alimentação escolar e estado nutricional de escolares no Nordeste e Sudeste do Brasil, 1997. Cad Saude Publica 2007; 23(5):1217-1226.

19. Martino HSD, Ferreira AC, Pereira CNA, Silva RR. Avaliação antropométrica e análise dietética de pré escolares em centros educacionais municipais no sul de M inas Gerais. Cien Saude Colet [periódico na internet] 2010 jan. [acessado 2010 jan 25]; [cerca de 10 p.]. Disponível em: http://www. cienciaesaudecoletiva. com.br

20. Fundo Nacional de Desenvolvimento da Educação (FNDE). Histórico. [acessado 2010 jan]. Disponível em: http://www.fnde.gov.br/index.php/ae-historico

21. DeM ayer E, Dallman P, Gurney JM, Hallberg L, Sood SK, Srikantia SG. Preventing and controlling iron deficiency anaemia through primary health care: a guide for health administrators and programme managers. Geneva: World Health Organization; 1989.

22. Brito DV. Estado nutricional de escolares de 7 a 11 anos de uma área suburbana de Recife. [dissertação]. Recife: Departamento de Nutrição da Universidade Federal de Pernambuco; 1982.

23. Veras ICL. Aspectos alimentares e nutricionais de escolares em educandários públicos do bairro da Várzea, Recife-PE. [tese]. Recife: Departamento de Nutrição da Universidade Federal de Pernambuco; 2004.
24. Lemos MCC. Estado nutricional de crianças, adolescentes e adultos no contexto da transição epidemiológica. [tese]. Recife: Departamento de Nutrição da Universidade Federal de Pernambuco; 2007.

25. American Society for Clinical Pathology (ASCP). Clinical applications of flow cytometry. ASCP National M eeting: Spring; 1990.

26. Programa das Nações Unidas para o Desenvolvimento (PNUD). Atlas de desenvolvimento humano no Recife. 2005. [acessado 2009 mar]. Disponível em: http://www.pnud.org.br/publicacoes/atlas recife/atlas recife idh bairros.pdf

27. Heijblom GSS, Santos LM P. Anemia ferropriva em escolares da primeira série do ensino fundamental da rede pública de educação de uma região de Brasília, DF. Rev Bras Epidemiol 2007; 10(2):258-266.

28. Santos M M, Diniz AS, Nogueira N N. Concentrações de hemoglobina e ferritina sérica em escolares da rede pública municipal de Teresina, Piauí, Nordeste do Brasil. Rev Bras Matern Infant 2008; 8(4):419-426.

29. World Health Organization (WHO). Worldwide prevalence of anaemia 1993-2005: WHO global database on anaemia. Geneva: World Health Organization; 2008.

30. Levy-Costa RB, Sichieri R, Pontes NS, Monteiro CA. Disponibilidade domiciliar de alimentos no Brasil: distribuição e evolução (1974-2003). Rev Saude Publica 2005; 39(4):530-540.

31. Queiroz AR, Szarfarc SC, M archiondi DML. A fortificação das farinhas de trigo e de milho no fornecimento de ferro para a merenda escolar. Nutrire 2008, 33(2):63-73.

32. Yip R. Significance of an abnormally low or high hemoglobin concentration during pregnancy: special consideration of iron nutrition. Am J Clin Nutr 2000; 72(Suppl.):272S-279S.

33. Bresani CC, Souza Al, Batista Filho M, Figueiroa JN. Anemia e ferropenia em gestantes: dissensos de resultados de um estudo transversal. Rev Bras Saúde M ater Infant 2007; 7:15S-21S.

34. Santos $C D$, Santos LM P, Figueiroa JN, M arroquim PM G, Oliveira MAA. Anemia em escolares da primeira série do ensino fundamental da rede pública de M aceió, Alagoas, Brasil. Cad Saude Publica 2002; 18(6):1757-1763.

Artigo apresentado em 15/07/2009

Aprovado em 27/01/2010

Versão final apresentada em 15/02/2010 